\title{
Methodology for Supply Chain Integration: A Case Study in the Artisan Industry of Footwear
}

\author{
Jorge Raúl Pérez-Gallardo, ${ }^{1,2}$ Beatriz Hernández-Vera, ${ }^{2}$ \\ Constantino Gerardo Moras Sánchez, ${ }^{2}$ Alberto Alfonso Aguilar Lasserre, ${ }^{2}$ \\ Rubén Posada-Gómez, ${ }^{2}$ Ulises Juárez-Martínez, ${ }^{2}$ \\ and Giner Alor-Hernández ${ }^{2}$ \\ ${ }^{1}$ Université de Toulouse, INPT, UPS, Laboratoire de Génie Chimique, 4 allée Emile Monso, 31432 Toulouse, France \\ ${ }^{2}$ Division of Research and Postgraduate Studies, Instituto Tecnológico de Orizaba, 852 Oriente 9, 94320 Orizaba, VER, Mexico
}

Correspondence should be addressed to Beatriz Hernández-Vera; bh.vera@hotmail.com

Received 11 April 2014; Accepted 20 June 2014; Published 7 September 2014

Academic Editor: Ker-Wei Yu

Copyright (C) 2014 Jorge Raúl Pérez-Gallardo et al. This is an open access article distributed under the Creative Commons Attribution License, which permits unrestricted use, distribution, and reproduction in any medium, provided the original work is properly cited.

\begin{abstract}
The supply chain is a major issue in a global market. The efficient relationship between supplier-producer-retailer conforms the perfect combination to achieve a high level of competitiveness according to the needs of increasingly demanding and changing markets. The difficulty of synchronizing the members within the SC is related to the inherent uncertain factors. This paper proposes a methodology for integrating the supply chain that can be applied in any type of business. To demonstrate its relevance, a case study is performed at a handmade shoe company. An improved demand forecasting, an optimal balanced production line, a proper inventory management of raw materials, and an efficient calculation of the delivery time to the customer represent some of the main results.
\end{abstract}

\section{Introduction}

Proper integration of supply chain (SC) is a major issue because in a global world the company competitiveness is the key to stay in the market. Nevertheless, in the competition for its permanence, companies must sail endless problems.

These problems force the enterprises not only to efficiently coordinate their activities internally, but also to establish and preserve harmonious relations with its suppliers and retailers within the SC. The synchronization of the supply chain is a topic that has been widely discussed in different approaches. A healthy relationship between the three main members (supplier-producer-retailer) leads companies to get a high level of competitiveness. The complexity of harmonizing these three elements lies in the various viewpoints and approaches that each member may have in order to generate an adequate coordination. For the manufacturer it is important to have a system that supports an efficient planning, scheduling, and production control, a proper selection of their suppliers and to define efficient distribution protocols in order to be able to integrate, synchronize, and optimize the performance of the entire chain.

The competitiveness of markets has forced manufacturers to select the most appropriate supply chain network to reduce total costs and loss of time. In the literature there are examples of various methodologies to achieve the supply chain integration, for example, Ghasimi et al. [1] used genetic algorithms to optimize a novel mathematical model for the supply chain network for defective products, in order to minimize the production, distribution, and maintenance costs. The model permits to determine the amount of economic production, the length of each cycle, and the number of defective products, waste products, and retail scarcity using just-in-time technique.

Efendigil and Onüt [2] present a methodology for SC integration from customers to suppliers through warehouses, retailers, and plants via both adaptive network based fuzzy inference system and artificial neural networks approaches. 
The methodology presented provides this integration by finding the requested supplier capacities using the demand and order lead time information across the whole SC in an uncertain environment. The sensitivity analysis is made by comparing the obtained results with the traditional statistical techniques.

Finally Hon and $\mathrm{Ng}$ [3] propose a Process Integration (PI) approach to supply chains, especially developing further the analogy between Pinch Analysis and the field of operational research. Their work provides clear insight into how the PI concept brings a significant contribution to the development of supply chain design.

In Mexico, according to the National Institute of Statistics, Geography and Informatics (INEGI) [4], over $90 \%$ of business units across the country are SMEs, which represent $52 \%$ of GDP. Most companies have seen a decline of their profits due to poor internal organization and administration. In addition, many of the products or goods are unattractive to customers. There is a fierce competition both national and international to find a distinctive feature that makes a product or service unique. One of the industries where the difficulties are more visible is the shoe industry. In this article the case of a shoe maker company is presented to demonstrate the performance of the methodology proposed.

We present a state of art in the following section, the problem to address will be exposed in the third section, in the fourth section the proposed methodology for the integration of supply chains is presented. In the fifth section the methodology is applied in a case study and finally the conclusions about the work are presented.

\section{State of the Art}

At the present, the highly competitive and changing markets have caused that companies demand quick responses that represent an advantage to face its competitors [5]. Besides the rapid evolution of technological innovation, the stiff competition and the fluctuating consumer demands have forced to establish a closer cooperation between suppliers, producers, and distributors, as well as the study of the relationships between the members within the chain supply in order to improve their performance [6].

Global competition is imposing to current industry to make high quality products in a faster and cheaper way. The demand for a quick exchange of information has increased, especially among remote sites, for creating a better cooperation and coordination. Several papers seek the effective coordination between the entities that make up the supply chain network. Traditionally, the strategic management of human resource had been focused as an isolated entity. Lengnick-Hall et al. [7] present a support framework for identifying factors that influence the efficient orientation of the supply chain and define strategic management practices to capitalize this orientation. The framework also generates a better human resource strategic management, a better relationships both internal and external at the organization, and understanding of the links among the human resource systems, the orientation of the supply chain, and the strategic results.
Palma-Mendoza et al. [8] propose a detailed plan to support the supply chain integration based on the unified framework of three different domains: redesign of business process, redesign of the supply chain process, and design e-business, which can be applied to any company or sector.

Several tools and techniques had been used to achieve the supply chain integration depending on the current needs. Eliman and Dodin [9] propose a supply chain integration as a network of projects that covers certain activities: sending, processing, and receiving orders. This network is modeled by mixed integer programming that captures various advantages and disadvantages as the stationary and transient inventory costs, processing activity costs, the shipping cost, and the penalty for delay in delivery of customer orders. The optimal solution found provides cycle times, modes of transport, processing times, and due dates at minimal cost. From another perspective Jafar [10] studied the supply chain in two stages in order to reduce the delivery time. He involved the distributors to participate in the coordination plan making emphasize in the importance of more reliable shipping equipments.

Mehdi [11] presents a programming model for multiple objectives with the purpose to adopt an integrated approach that support the decision making on the optimal allocation of the limited resources in the supply chain, the suppliers selection, the production, distribution, and schedule of supplies, and the lower cost and the higher income, thus, maximizing the benefit of the chain. The model considers the integration level of suppliers, producers, and distributors, as well as the free relationships among the producers to manage the product supply through the process or even the products of each other, and finally consider the effect of the economic factors in decisions such as inflation.

Zhang et al. [12] conducted a biobjective model for the design of the supply chain for dispersed manufacturing considering essential trade-offs between the supply chain costs and the lead time to determine the optimal location of the manufacturing activities.

It is imperative for contemporary companies proactively seek to improve continuously the performance of their supply chain. The coordination and the integration of the decision making across the supply chain between the various partners that conform it are often used for this purpose. Such coordination strategies of the supply chain include the use of a common cycle time, fix quantity discounts, the use of an optimal batch sizing, the quality improvements and inspections, to mention a few. An important issue in the supply chain refers to the incorporation of human factors presented thought the supply chain task such as the failed quality inspections and production improvements due to learning. Khan et al. [13] present a simple mathematical model for determining an optimal inventory policy seller-buyer that considers the quality inspection errors. The objective is to minimize the annual cost incurred in the supply chain.

To ensure the effective supply of biomass to produce biofuels on a large scale, Tao et al. [14] presented an optimization model of the supply chain to minimize the annual costs of biomass-ethanol production by optimizing both strategic and tactical planning decisions simultaneously. The mixed integer linear programming model optimizes 
the activities like biomass collection, packaging, transportation, internal transport, preprocessing, storage, production, and distribution of ethanol. The locations, capacities and quantity of facilities and distribution patterns of biomass and ethanol are considered as key strategic decisions, while biomass production, delivery and operation schedules and inventory monitoring are key tactical decisions.

A common problem at network design for integrated supply chain consists in determinate the locations of distribution centers and the allocation of customers and suppliers to the centers of distribution. The problem tries to seek at the same time the distribution of products from the manufacturer to the customer and the collection of components from suppliers to manufacturers through crossdocking at the distribution centers. Zhang et al. [15] propose an algorithm based on Lagrangian relaxation which includes the co-location of different types of distribution centers and the coordination of the transport to achieve savings in transportation costs.

Eren et al. [16] propose an integrated model that jointly optimizes the strategic and tactical SC closed loop decisions. Strategic level decisions concern the amounts of goods flowing in chains downstream and upstream, while tactical level decisions concern the balancing of removing lines of inverse chain. The objective is to minimize transportation, purchases, renovation, and operation costs of disassembly workstations. Finally, the authors formulated and described a nonlinear mixed integer programming model for this situation.

Sarkar and Majumder [17] propose an integrated SC supplier-seller model. Two models constructed on the basis of the probability distribution of the time were presented. The waiting time is normally distributed in the first model, while in the second model a free distribution for the delivery time is considered. For this second model, only the mean and standard deviation are known. The objective of the model is to reduce the total cost of the system taking into account the reduction of installation costs for the supplier.

A manufacturing SC with multiple suppliers in the presence of multiple uncertainties as uncertain supplies of materials, stochastic times of production and randomly costumer demand is a scenario that Song et al. [18] addressed in their study. They formulated an inventory management policy, a raw materials acquisition policy, and a production control policy using the stochastic dynamic programming approach. Then they investigated other strategies as the reduction of suppliers and the differentiation of suppliers under the integrated inventory management policy, establishing qualitative relationships between the amount of suppliers, the supplier capabilities, and the total expected cost. The model allows achieving quantitatively the best trade-off between reducing suppliers and improving the supplier capability, in addition to quantifying the supplier differentiation in purchase decisions.

The multiskill environment is a problem for staff allocation, so Telhada [19] formulates a mixed integer programming model whose application supports the assignment of shifts and tasks on the same problem.

Finally Zhang [20] formulated a model and a method for the coordination of production and inventory cycles throughout the manufacturing SC involving reverse logistics for items with multiperiod finite horizon inventory. An entire manufacturing SC involving reverse logistics includes category 2 Suppliers, category 1 suppliers, manufacturers, distributors, consumers, and Recycling. The authors propose a mathematical model to represent the behavior of this system; the solution methods proposed are based on a decentralized decision-making process and a combination of decentralized and centralized decision, known as the semicentralized decision making process. The centralized decision-making process is achieved by a mixed integer linear programming.

This brief literature review allows seeing the different angles from which the SC has recently been addressed. In general it can be concluded that the above efforts are looking to integrate the various links in the chain, in order to reduce costs and increase yields. But in particular, this study has dealt with the management and inventory control, the distribution of finished products, the identification of multiple suppliers in the presence of uncertainties, and the inclusion of human errors and their impacts to the chain, with this last point being the most important. The ability, performance, and proceess that the human resource had, can move the chain differently.

The above represents individual efforts to demonstrate how particular issues affect the operation of the SC. Also various techniques, algorithms or tools were used to potentially improve the SC and support the decision making process. All these contributions were tested into SC that does not include artisanal production processes, which opens the possibility to question the validity of these applications in such processes.

This article proposes a methodology for ensuring the SC integration regardless of the type of business, using traditional engineering techniques and artificial intelligence techniques. This integration does not detract from the traditional methods of problem solving but they complement each other.

\section{Problem Statement}

The SC synchronization is a topic that has been widely discussed in recent years as it could be shown in the previous section. Due to various factors, companies not only search the efficient coordination of their activities internally, but also maintain a harmonious relationship with its suppliers and dealers within this chain.

Globalization and new technologies are commonly terms used to reflect the current reality and are associated with ideas of change, speed, real-time communications, and electronic commerce, among others. However in this changing world still prevail situations such as the development of customized products for specific needs, the correct allocation of task in a process, and the reduction of delivery time to name a few.

At the present, company competitiveness is the key to staying in the market, but the fact is that in the competition for their permanence businesses must deal with endless problems. In addition some businessmen persist to see their companies as a standalone system and not as a member of a bigger system that influences the future behavior of the market. They do not consider that the competition 


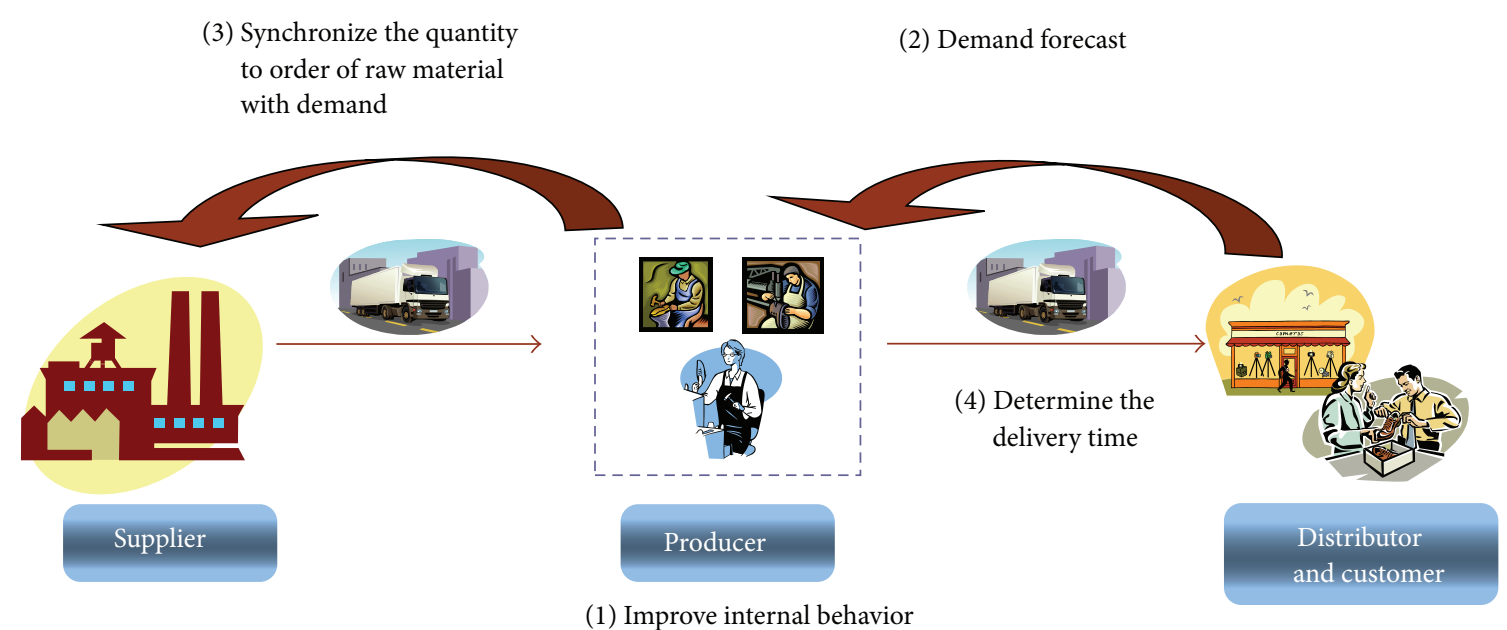

FIGURE 1: Methodology for the integration of the supply chain.

between networks and the integration of key processes with trading partners allow them to form a much larger system, where suppliers and customers are no longer part of its environment, but part of his whole system known as SC.

It is necessary for companies to have support tools that allow them to guide their efforts to identify their place within an efficient SC, establishing harmonious relations with suppliers and distributors, in order to establish itself in a competitive market.

That why this paper presents a methodology to integrate the SC that aims to be a tool to optimize the resources and the flow of certain products thoughout the SC and even more to demonstrate the use of the proposed methodology a case study in the artesian company of footwear is presented. In general, the integration is made in two levels: first, the internal processes of the footwear company are analyzed and the solution of possible problem that could block the SC integration are looked for; second, strategies that permit an integration with the suppliers and retailers are proposed and evaluated.

This methodology is a flexible tool that enables each of its steps apply both traditional engineering tools and sophisticated or specific tools. Finally, it is appropriate for any type of business, including those whose production process is artisanal.

\section{Methodology for Supply Chain Integration}

As we already mention all companies that conform the SC must interact in order to achieve competitive advantage, which represent the reason why a methodology to guide the integration of each of the links in the SC was formulated. Each step of the proposed methodology is described below and schematized in Figure 1.

(1) Improve internal behavior: the first step for the successful integration of the company with the others that take part of the SC is to improve internal performance by applying traditional techniques to diagnose system performance, setting goals and strategies to improve the indicators and assess the progress.

(2) Demand forecast: this is a critical step to integrate the SC. A good demand forecast achieves synchronization of all the companies that conform the chain. This information is provided by historical records of the main selling points.

(3) Synchronize the quantity to order of raw material with demand: the aim is to reduce the costs generated by the stock of raw materials but they must be available at the time which has to make use of it from the forecasted demand.

(4) Determine the delivery time: the aim is to establish reliable times or delivery of the final product represents the last point.

The four steps of the methodology together enable the company improve its internal performance and to establish a harmonious and synchronized relationship these changes will place the company in its respective SC and their respective partners within the same chain. The integration result of the application of the proposed methodology will allow companies able to face the rest of the factors promoting the competitiveness of enterprises.

\section{The Case of Study: Application of the Methodology for the Integration of the Supply Chain}

The footwear industry in Mexico is considered one of the most important in the artisanal field, with products whose quality is widely recognized. But unfortunately this industry like many others is submerged in a chronic crisis since several years. According to the National Chamber of the Footwear Industry in Mexico [21] the cost of production ranges between 18 and 20 dollars, while in Asian countries varies between 6 or 8 dollars. Although, some Mexican companies have made the effort to get new technology and 
maintain high levels of international competitiveness, it is virtually impossible to compete against countries like China due to their business strategies and their labor cost.

The case of a handmade shoe company located in Southeastern Mexico is exposed and for which the methodology proposed is applied in this paper in order to prove its effectiveness and allow the company to become more competitive.

The shoe company studied follows artisanal production processes. In this kind of companies, the rate of work depends on the capabilities and skills of workers. In addition, the lack of control over their inventory and delivery times which are key criteria for determining the competitiveness of any company makes almost impossible to get a successful integration of SC. That is why a correct distribution of the task in order to achieve the goal and objectives is fundamental.

The shoe company under study represents only one link in the network of SC in which it is immersed, and to become more competitive it must be integrated to the rest of the links.

5.1. Improve the Internal Behavior. The first step of the methodology is to improve the internal behavior and focusing mainly on the production of shoes, so we will seek to make more efficient this area. This first step is divided into three activities which are detailed below:

Step 1 (simulation of the production process). To better understand the operation of the system under study simulation was used to obtain information on the performance of certain actions and to define strategies to improve these indicators.

Step 2 (solution to the identified problem). After detecting the problem, the best alternative to solve it was looked for.

Step 3 (evaluation of proposed alternative). To prove if what is being proposed really solves the problem and improves the performance of the measure evaluates, the simulation model of the production area is modified. The statistical results obtained are compared. If the goal is not achieved, another solution is proposed and evaluated.

5.1.1. Simulation of the Production Process: Modeling and Analysis System. Discrete event simulation is a technique that allows developing computer models of some desired system, in order to identify unknown characteristics and/or conduct experiments in a defined time frame, respecting some imposed restrictions.

One of the initial conditions to successfully implement a correct SC management is to improve the efficiency of internal operations. The simulation model was focused on the analysis of the department in which according to the owner there are more problems.

The objectives pursued with the development of the simulation model were as follows:

(i) analyze the current production process for the 3 products (shoe models) with greater demand;

(ii) Analyze the productivity indicators for workers in the production process at the current system.
The department studied has 6 operators. The direction sets the goal of producing 250 pairs of shoes by day for any type of product with a transfer batch of 25 pairs of shoes, based on time study conducted by the Chamber of the Footwear Industry of the State of Guanajuato [21]. Operations were assigned according to the skills of each employee and no previous study of line balancing was performed. The main problem of the production system detected was the failure of workers to get the goal imposed during the working time journey, causing unpaid overtime. Another aspect is the existences of bottlenecks that cause the workers to have no work to do. When this happens, they have to support a worker who has a lot of inventory in batch process causing the transfer not to be respected and the bottleneck keeps changing.

A simulation model that represents the system as it currently operates was made. The model takes into account all the operations assigned to 6 workers for the manufacture of 3 products. The resulting simulation model allowed knowing some indicators such as the cycle time, the productivity of each worker, and machine as well as the main bottleneck.

(1) Data Collection and Definition of Current System Performance Parameters. The process flowchart, time study, the name of the workers, their skills, and their assigned tasks within the production process was analyzed to build the simulation model. Also it was necessary to know about the available machinery, the capacity and size of the machinery, and the total size of the production area to develop the layout.

To build the simulation model, the cutting tasks, the processing time, and the workers who are part of the die cutting department were took into account.

The following measures of performance were evaluated: the cycle time, the productivity of workers and machines, the bottlenecks, and the efficiency of line balancing.

(2) Construction and Verification of the Simulation Model. To build the simulation model ProModel, which is a powerful simulator with integrated animation, was used.

The model contains each of the elements of interest in the system, such as machines, pieces, and personnel. Figure 2 shows the layout of the model made.

Once the construction of the model was finished, we proceeded to verify it using the tracking system which ProModel has integrated. It shows each of the events every minute of time simulated, which allows a broad view of what is happening internally.

(3) Verification, Validation, and Experiment Design. The simulation model was validated in order to confirm that truly reflect reality, these phases include the pilot testing, the validation, and the experiment design.

Pilot tests were conducted to obtain preliminary information provided by the simulator. This information will serve for the later stages; in this case 10 pilot runs were performed.

Validation is the comparison of the observed data and the simulated data, in order to prove that the differences are statistically insignificant.

Statistical paired $t$-test was used to compare the results obtained from the simulation with real data. The time that 


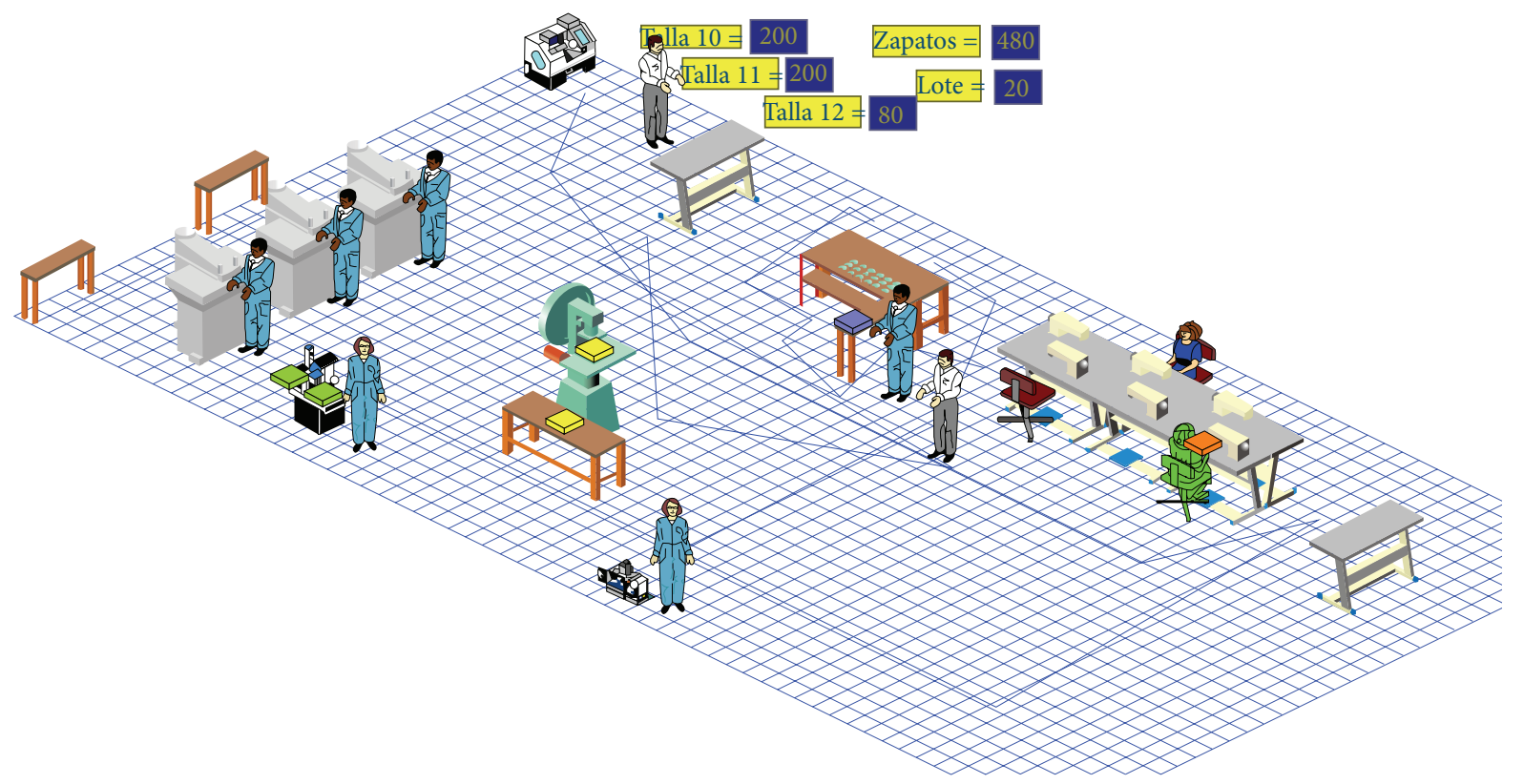

Figure 2: Model layout.

250 pairs of shoes for each of the three products takes to be produced was the performance measure. The results conclude that the difference observed between $\mu_{x}$ (mean of observed data) and $\mu_{y}$ (mean of simulated data) is not statistically significant at a confidence level of $95 \%$, and may be explained by random fluctuations, thereby the model was accepted.

Finally, to determine the optimal number of runs for the simulated model, the method of estimating the mean $\mu=E(x)$ with a specific error is used. In other words, if 100 independent confidence intervals are constructed of $90 \%$, is expected $\bar{X}$ to have an absolute error $\beta$ of at most 90 cases; 10 intervals around the absolute error would be greater to $\beta$.

The time that takes to produce 250 pairs of shoes in minutes, obtained from the 10 independent pilot runs were used and the average manufacturing time in minutes with an absolute error $\beta=5$ minutes and a confidence level of $99 \%$ was estimated. With this procedure, we found that the optimal number of replications was 14 runs.

(4) Analysis of Results. Results after running the model 14 times are shown in Table 1 . Table 1 shows the value of the three parameters selected: the total manufacturing time, manufacturing cycle (sum of durations of all the tasks assigned to each worker) and cycle time for each of the three shoes products. Finally the individual productivity and efficiency of the whole production line based on a production rate of 250 pairs of each of the three products is included.

The results show that the actual efficiency of the production line on average is 56\%; this represents the percentage of time that the resources of the factory are working, while the remaining $44 \%$ is considered idle time. It also states that staff's productivity is uneven. While the worker 6 is occupied $23 \%$ of the total simulation time, the worker 2 has nearly an $89 \%$ of total time occupied. This means that the workload is poorly distributed and does not justify the hiring of new staff. A new task assignation is required.
TABLE 1: Results of the simulation model of the current manufacturing process.

\begin{tabular}{lcc}
\hline Parameter & $\begin{array}{c}\text { Mean } \\
\text { (minutes) }\end{array}$ & $\begin{array}{c}\text { Standard deviation } \\
\text { (minutes) }\end{array}$ \\
\hline $\begin{array}{l}\text { Total time for manufacturing } \\
\text { three products }\end{array}$ & 1252.4 & 5.9 \\
Product 1 & & \\
$\quad$ Manufacturing cycle & 591.4 & 7.2 \\
$\quad$ Cycle time (batch) & 22.0 & 0.2 \\
Product 2 & & \\
$\quad$ Manufacturing cycle & 469.8 & 3.0 \\
$\quad$ Cycle time (batch) & 21.0 & 0.2 \\
Product 3 & & 3 \\
$\quad$ Manufacturing cycle & 457.0 & 3.0 \\
$\quad$ Cycle time (batch) & 21.0 & 0.1 \\
\hline Parameter & Mean & Standard deviation \\
Workers & $(\%)$ & $(\%)$ \\
$\quad$ Worker 1 & & \\
Worker 2 & 63.3 & 0.4 \\
Worker 3 & 88.9 & 0.1 \\
Worker 4 & 74.2 & 0.5 \\
Worker 5 & 61.2 & 0.4 \\
Worker 6 & 46.8 & 0.8 \\
\hline Line efficiency & 23.6 & 0.1 \\
\hline
\end{tabular}

5.1.2. Optimization of Supply Chain. The results of the simulation showed that there is low productivity of both workers and machines. Likewise, we emphasize that the lead time is too high. Because of this situation the perfect balance at the production line is necessary, that is, the sum of the operation 
time allocated to each operator must be equal to the cycle time and, therefore, the product flows without delays.

(1) Balancing Production Lines. Following the proposed methodology (Section 5.1), we propose the line balancing that consists of assigning tasks to individual operators or workstations so that any performance measure is optimized.

The problem of balancing the manufacturing lines of shoes should considers the following.

(a) Operation times are probabilistic.

(b) The tasks cannot be executed in an arbitrary order given the precedence requirements.

(c) It is desired to minimize the number of workers.

(d) The line is balanced with the available machinery.

(e) The tasks cannot be divided between two or more stations.

(f) The setup times are negligible.

(g) Each station processes only one task at a time.

(h) All tasks must be processed once.

(i) Transportation time between a work stations is not considered

(j) The tasks run only once for a season (considered a serial line).

(k) Some workers cannot perform certain tasks.

The variables used to construct the model of line balancing are as follows:

$k=$ Total number of workstations

$n=$ Total number of manufacturing processes

$c=$ Cycle time

$t_{i}=$ Manufacturing time operation $j, j=1, \ldots, n$

$I=$ Idle time of the production system

$X_{i j}=\left\{\begin{array}{l}1, \text { If the process } j \text { is assigned to workstation } i, i=1, \ldots, k \\ 0, \\ \text { otherwise. }\end{array}\right.$ by (1)

The objective function is to minimize the idle time given

$$
I=k c-\sum_{j=1}^{n} t_{j} .
$$

This function is subject to the following restrictions:

$$
\begin{gathered}
\sum_{j=1}^{n} X_{i j} t_{j} \leq c, \quad i=1,2, \ldots, k, \\
\sum_{i=1}^{k} X_{i j}=1, \quad j=1,2, \ldots, n, \\
X_{h v} \leq \sum_{i=1}^{h} X_{i u}, \quad h=1,2, \ldots, k ;(u, v) \in R,
\end{gathered}
$$

where the total time of all processes assigned to each workstation must not exceed the cycle time (2), a task can only be assigned to a workstation, (3) and precedence relationships exist (4). The precedence relationships were modeled as published by Ragsdale and Brown [22].

Most of the operations for the manufacture of shoes in this company are manual; because of this, the operating time of some activity varies from one shoe to another so each time operation follows a probabilistic distribution at the mathematical model. Each operation must be assigned to only one worker; this situation is represented by a binary variable with value 1 when the process $j$ is assigned to workstation $i$ and 0 otherwise. We proposed to optimize the lines balancing problem by a simple genetic algorithms.

\section{(1) Solution of the Mathematical Model}

(i) Optimization of the Mathematical Model through a Genetic Algorithm (GA). The objective function was minimizing the idle time. As long as the idle time decreases the efficiency of the line increases. The efficiency of the line is considered as percentage, thus the optimal value is $100 \%$.

The basic structure of classical Assignment Problem (AP) is the minimization of an objective function involving cost or time. It is a well-studied problem in the optimization literature due to its many applications such as facility location, personnel scheduling, task assignment, and so forth. It is formulated as an 0-1 optimization problem. However, many real-life problems consider constraint such as budgetary limitations, time restrictions, or personal abilities, and so forth, that affect the assignment decisions. Due to this fact, finding a solution becomes more complex. Many authors solve this type of problems by genetic algorithm with excellent results $[23,24]$.

The genetic algorithm is a useful adaptive method to solve search and optimization problems. Based on the theory of Darwinian evolution, it is a robust technique that can solve problems in various areas including those in which the application of other methods is difficult. While these algorithms do not guarantee an optimal solution to the problem is obtained, empirical evidence shows that it can find solutions to an acceptable level in a competitive time compared to other combinatorial optimization algorithms. The assignment problem for the case of the proposed study is a clear consequence of the same situations. The Simple Genetic Algorithm used, also called Canonical, was encoded based on the binary variable that assign the task to its respective workstation. Genetic Algorithm (GA) includes three fundamental genetic operations: selection, crossover, and mutation. The first step in any GA is to generate an initial population with a group of individuals randomly created. The individuals in the population are then evaluated and assigned a fitness value. The evaluation function is provided by the operator and gives the individuals a score based on how well they perform at the given task (fitness value). Two individuals are then selected based on their fitness; the higher the fitness, the higher the chance of being selected.

After selection has been carried out, crossover is applied to randomly paired individuals. The recombined individuals create one or more off-spring. This can be viewed as creating the new population. The randomly mutation of the offspring is then applied. In terms of GAs, mutation means a random change of the value or a gene in the population. After 
TABLE 2: Comparison of the results obtained after line balancing according to the modification of the simulation model.

\begin{tabular}{|c|c|c|c|c|}
\hline \multirow{2}{*}{ Parameter } & \multicolumn{2}{|c|}{ Unbalanced system } & \multicolumn{2}{|c|}{ Balanced System } \\
\hline & Mean (minutes) & Standard deviation (minutes) & Mean (minutes) & Standard deviation (minutes) \\
\hline $\begin{array}{l}\text { Total manufacturing time } \\
\text { (three products) }\end{array}$ & 1252.4 & 5.9 & 1121.1 & 7.2 \\
\hline \multicolumn{5}{|l|}{ Product 1} \\
\hline Manufacturing cycle & 591.4 & 7.2 & 550.3 & 4.2 \\
\hline Cycle time (batch) & 22.0 & 0.2 & 20.1 & 0.1 \\
\hline \multicolumn{5}{|l|}{ Product 2} \\
\hline Manufacturing cycle & 469.8 & 3.0 & 417.5 & 2.4 \\
\hline Cycle time (batch) & 21.0 & 0.2 & 19.8 & 0.1 \\
\hline \multicolumn{5}{|l|}{ Product 3} \\
\hline Manufacturing cycle & 457.0 & 3.0 & 392.0 & 1.8 \\
\hline Cycle time (batch) & 21.0 & 0.1 & 19.0 & 0.2 \\
\hline Parameter & Mean (\%) & Standard deviation (\%) & Mean (\%) & Standard deviation (\%) \\
\hline \multicolumn{5}{|l|}{ Workers } \\
\hline Worker 1 & 63.3 & 0.4 & 81.5 & 0.5 \\
\hline Worker 2 & 88.9 & 0.1 & 66.6 & 0.5 \\
\hline Worker 3 & 74.2 & 0.5 & 67.9 & 0.6 \\
\hline Worker 4 & 61.2 & 0.4 & 57.1 & 0.4 \\
\hline Worker 5 & 46.8 & 0.8 & 66.6 & 0.5 \\
\hline Worker 6 & 23.6 & 0.1 & 64.5 & 0.5 \\
\hline Line efficiency & 56.0 & & 73.6 & \\
\hline
\end{tabular}

the process of selection, recombination and mutation, the next population can be evaluated. The process continues until a suitable solution has been found or when a given number of generations have been reached.

GA parameters used are population size 200 individuals, 400 generations (stopping criterion), $40 \%$ crossover rate, and mutation rate of $30 \%$. A new allocation of tasks for each of the three products (different models of shoes) separately was generated.

(2) Evaluation of Proposed Alternative. With the new tasks configuration for each products is necessary to make changes to the simulation model to verify that this configuration reduces manufacturing time and achieves a proper production line balancing between existing workstations. This corresponds to Step 3 (improvement rate) of the proposed methodology.

The results show an increase in the efficiency of the line of $17.5 \%$, Table 2 shows a comparison of the current system and the system after balancing it.

To determine whether this reduction in manufacturing time is significant, a statistical test between means of two small samples was completed. It was concluded with $95 \%$ confidence that the proposed improvements will cause a reduction in the cycle time was performed.

5.2. The Demand Forecast. Step 2 of the methodology is the demand forecasting. To forecast the demand and calculate the monthly cycle time, historical record of the sale was used.
To determine the type of forecast to use it was necessary to plot the demand data. As shown in Figure 3, the behavior of the demand for each pattern is irregular, therefore has no tendency, or seasonality. To test this assumption the nonparametric statistical test of Daniels was performed to detect the seasonality of a series. This statistical test does not require the assumption of normality in the population. It was concluded to a $95 \%$ confidence that the monthly demand for shoes has not trend.

Once it was found that a model without trend is the most appropriate, the next step was to select the appropriate procedure to fit the data to the model. Methods of exponential smoothing, moving averages and updated mean were applied to forecast the demand for each of the selected shoe's models. For each product the best forecasting model was selected based on the performance of the three measures of statistical adequacy (median absolute deviation, MAD; root mean square error, RMSE; and mean absolute percentage error, MAPE).

5.2.1. Adjusting the Forecast by Fuzzy Logic. Deterministic forecasting methods have as a principle that every event is determined by the chain of cause and consequence, regardless of random events.

In the case study, to determine the demand it was convenient to use a fuzzy logic model, since it allows making decisions based on uncertain data or subjective knowledge, which are usually presented through linguistic variables. In this research, the fuzzy logic model proposed by Escobar 


\begin{tabular}{cccc}
\hline Month & Product 1 & Product 2 & Product 3 \\
\hline February & 5696 & 4386 & 2807 \\
March & 8462 & 5416 & 4387 \\
April & 5971 & 4837 & 3482 \\
May & 9400 & 5640 & 4343 \\
June & 8206 & 6565 & 3939 \\
July & 5771 & 4155 & 3324 \\
\hline
\end{tabular}

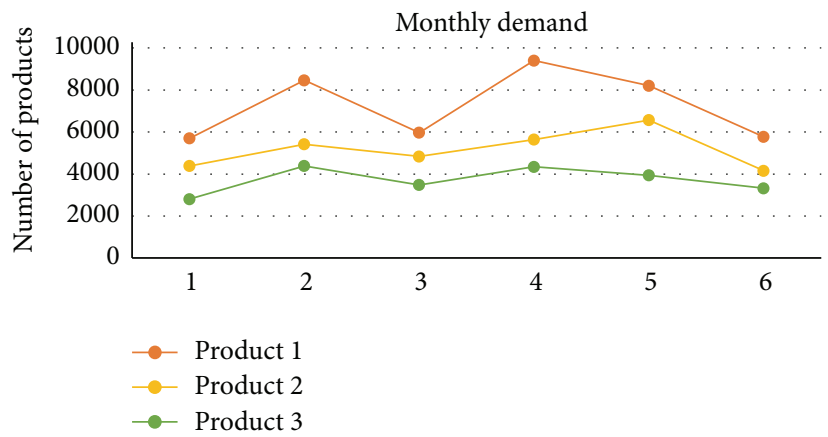

FIGURE 3: Graph of the monthly demand.

Gómez [25] was applied. This is still part of Step 2 of the methodology.

(1) Linguistic Variables and Fuzzy Sets. Fuzzy logic is an artificial intelligence technique that allows modeling situations whose variables are measured according to a subjective scale, or that depend on knowledge or opinion of experts in the subject concerned and also arise under uncertain conditions. The process involves identifying some variables, defined as input, which directly influences another variable, identified as output and their respective levels of response. The result will be a model that is able to translate subjective information on reliable and easily quantifiable results.

This model considered three factors which have influence demand according to expert knowledge: season, perception, and offer. These factors are described below.

Season as linguistic variable is the expression that refers to the behavior of demand in a given period compared to the same period of previous years. Three output cases were considered representing three fuzzy sets for this variable: low, medium and high season.

The membership functions for each of the fuzzy sets are shown in (5) as follows:

$$
\begin{gathered}
\mu_{\text {Low }}(\text { Season })= \begin{cases}1 ; & W \leq A, \\
1-\frac{B-W}{B-A} ; & A<W \leq B, \\
0 ; & B \leq W,\end{cases} \\
\mu_{\text {Medium }}(\text { Season })= \begin{cases}0 ; & W \leq A, \\
1-\frac{B-W}{B-A} ; & A<W \leq B, \\
1-\frac{W-B}{C-B} ; & B<W<C, \\
0 ; & C \leq W,\end{cases} \\
\mu_{\text {High }}(\text { Season })= \begin{cases}1 ; & W \leq B, \\
1-\frac{C-W}{C-B} ; & B<W \leq C, \\
1 ; & C \leq W .\end{cases}
\end{gathered}
$$

Perception as a linguistic variable is defined as the feeling of satisfaction that the customer has because the product met their expectations and needs. Fuzzy sets that are part of this variable are three: poor, fair, and good perception. Membership functions are shown in (6) as follows:

$$
\begin{aligned}
& \mu_{\text {Poor }}(\text { Perception })= \begin{cases}1 ; & X \leq A^{\prime}, \\
1-\frac{X-A^{\prime}}{B^{\prime}-A^{\prime}} ; & A^{\prime}<X<B^{\prime}, \\
0 ; & B^{\prime} \leq X,\end{cases} \\
& \left.\mu_{\text {Fair }} \text { (Perception }\right)= \begin{cases}1 ; & X \leq A^{\prime}, \\
1-\frac{B^{\prime}-X}{B^{\prime}-A^{\prime}} ; & A^{\prime}<X<B^{\prime}, \\
1 ; & B^{\prime} \leq X \leq C^{\prime}, \\
1-\frac{X-C^{\prime}}{D^{\prime}-C^{\prime}} ; & C^{\prime}<X<D^{\prime}, \\
0 ; & D^{\prime} \leq X,\end{cases} \\
& \mu_{\text {Good }}(\text { Perception })= \begin{cases}0 ; & X \leq C^{\prime}, \\
1-\frac{D^{\prime}-X}{D^{\prime}-C^{\prime}} ; & C^{\prime}<X<D^{\prime}, \\
1 ; & D^{\prime} \leq X .\end{cases}
\end{aligned}
$$

Offer as linguistic variable is defined as the level of competition that exists for the product analyzed at the market. For this variable three output alternatives were defined as fuzzy sets and are as follows: the level of competition is little, medium or large. The membership functions are presented in (7) as follows:

$$
\begin{aligned}
& \mu_{\text {Little }}(\text { Offer })= \begin{cases}1 ; & Y \leq A^{\prime \prime}, \\
1-\frac{Y-A^{\prime \prime}}{B^{\prime \prime}-A^{\prime \prime}} ; & A^{\prime \prime}<Y \leq B^{\prime \prime}, \\
0 ; & C^{\prime \prime} \leq Y,\end{cases} \\
& \mu_{\text {Medium }}(\text { Offer })= \begin{cases}0 ; & Y \leq A^{\prime \prime}, \\
1-\frac{B^{\prime \prime}-Y}{B^{\prime \prime}-A^{\prime \prime}} ; & A^{\prime \prime}<Y \leq B^{\prime \prime}, \\
1-\frac{Y-B^{\prime \prime}}{C^{\prime \prime}-B^{\prime \prime}} ; & B^{\prime \prime}<Y<C^{\prime \prime}, \\
0 ; & C^{\prime \prime} \leq Y,\end{cases} \\
& \mu_{\text {Large }}(\text { Offer })= \begin{cases}0 ; & Y \leq B^{\prime \prime}, \\
1-\frac{C^{\prime \prime}-Y}{C^{\prime \prime}-B^{\prime \prime}} ; & B^{\prime \prime}<Y \leq C^{\prime \prime}, \\
1 ; & C^{\prime \prime} \leq Y .\end{cases}
\end{aligned}
$$



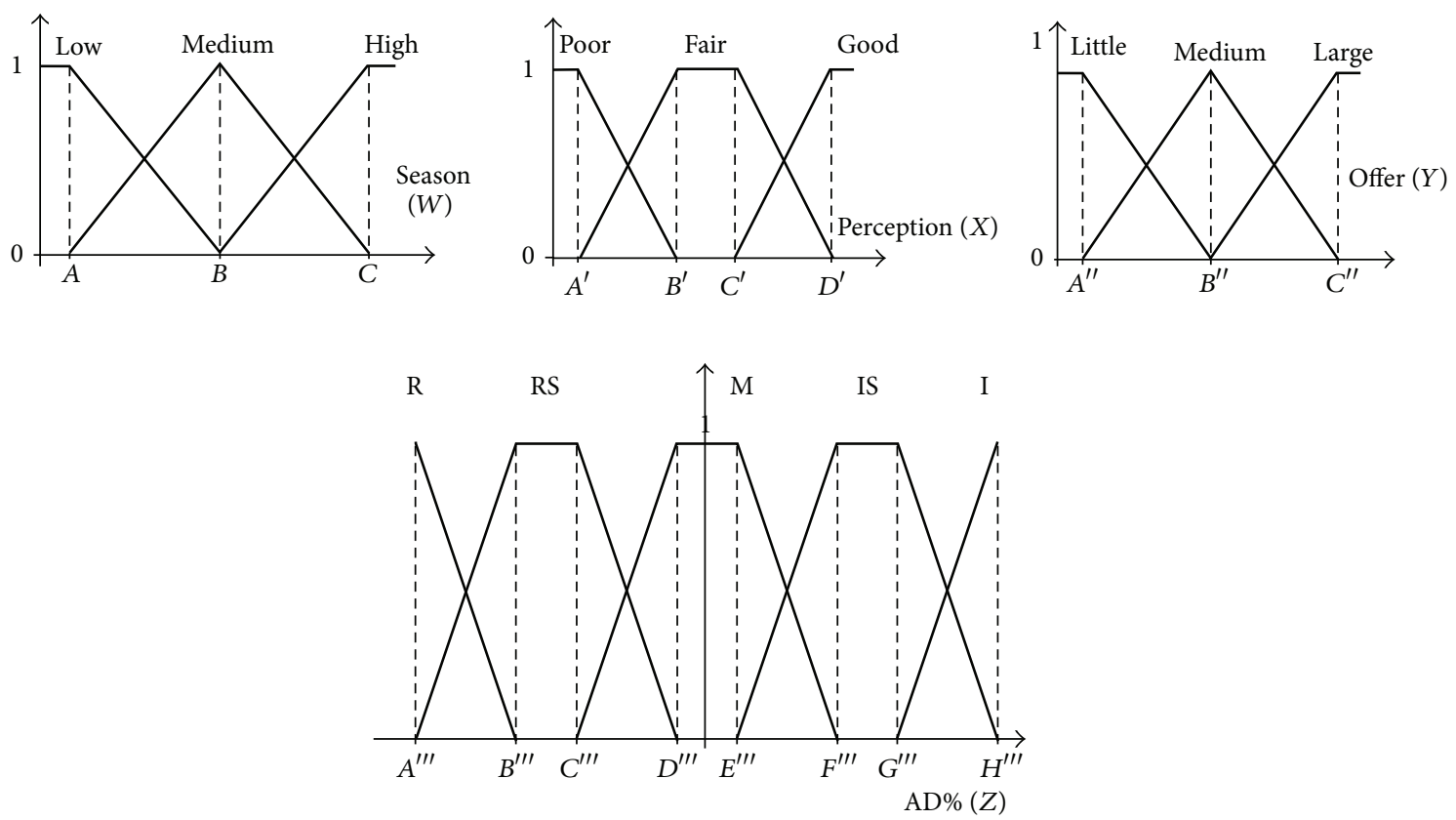

FIGURE 4: Graphical representation of the fuzzy sets of the linguistic input variables and output AD.

TABLE 3: Fuzzy rules base.

\begin{tabular}{|c|c|c|c|c|c|c|c|c|c|}
\hline & \multicolumn{9}{|c|}{ Perception } \\
\hline & \multicolumn{3}{|c|}{ Poor } & \multicolumn{3}{|c|}{ Fair } & \multicolumn{3}{|c|}{ Good } \\
\hline & \multicolumn{3}{|c|}{ Offer } & \multicolumn{3}{|c|}{ Offer } & \multicolumn{3}{|c|}{ Offer } \\
\hline & Little & Medium & Large & Little & Medium & Large & Little & Medium & Large \\
\hline \multicolumn{10}{|l|}{ Season } \\
\hline Low & $\mathrm{R}$ & $\mathrm{R}$ & $\mathrm{R}$ & RS & $\mathrm{R}$ & $\mathrm{R}$ & M & M & RS \\
\hline Medium & $\mathrm{M}$ & RS & $\mathrm{R}$ & IS & $\mathrm{M}$ & RS & I & IS & M \\
\hline High & $\mathrm{M}$ & RS & $\mathrm{R}$ & IS & IS & $\mathrm{M}$ & I & I & IS \\
\hline
\end{tabular}

Therefore, the output variable of the model is Adjusted Demand and is defined as the change in the level of the projection of the aggregate demand percentage. Under this consideration five cases are presented, which allow to define the fuzzy sets belonging to this: reduce, reduce slightly, maintain, increase, and increase slightly (8). Consider

$$
\begin{aligned}
& \mu_{\text {Reduce }} \text { (Adjusted demand) } \\
& = \begin{cases}0 ; & Z<A^{\prime \prime \prime}, \\
1-\frac{Z-A^{\prime \prime \prime}}{B^{\prime \prime \prime}-A^{\prime \prime \prime}} ; & A^{\prime \prime \prime} \leq Z<B^{\prime \prime \prime}, \\
0 ; & B^{\prime \prime \prime} \leq Z .\end{cases}
\end{aligned}
$$

Both the input variables and the output variable, in this image, are presented in Figure 4 which shows the relationships described in the membership functions ((5)-(8)) where the different scenarios that can occur for each variable are clear, and allows modeling these variables by geometric figures.

(2) Fuzzy Rules. Given the input linguistic variables and their fuzzy sets (3 variables with three sets each) a base of 27 fuzzy rules was obtained. In this set of rules the knowledge of the experts consulted on decision-making related to the adjustment of the forecast demand is summarized. The summary of the rules used for the inference process is presented in Table 3.

Where D means high projected demand reduction, DP means low projected demand reduction, $M$ maintains the projected demand, IP means low projected demand increase, and finally I means high projected demand.

The defuzzification process was the next step. This process consists in obtaining the values through fuzzy membership functions of the values assigned to the input variable. In this model the degree of membership in fuzzy sets was obtained by evaluating the values assigned to linguistic variables using the membership functions. If, after applying the fuzzy equations the value obtained for a given set is greater than zero, it is considered to belong to the set analyzed with a degree equal to the value found. Otherwise, it is considered that the degree of belonging to the set is zero.

The fuzzy logic model applied to the demand of each of the products is shown in Tables 4, 5, and 6. This modification caused the forecasting model fit better to actual demand. 
TABle 4: Adjusted demand product 1.

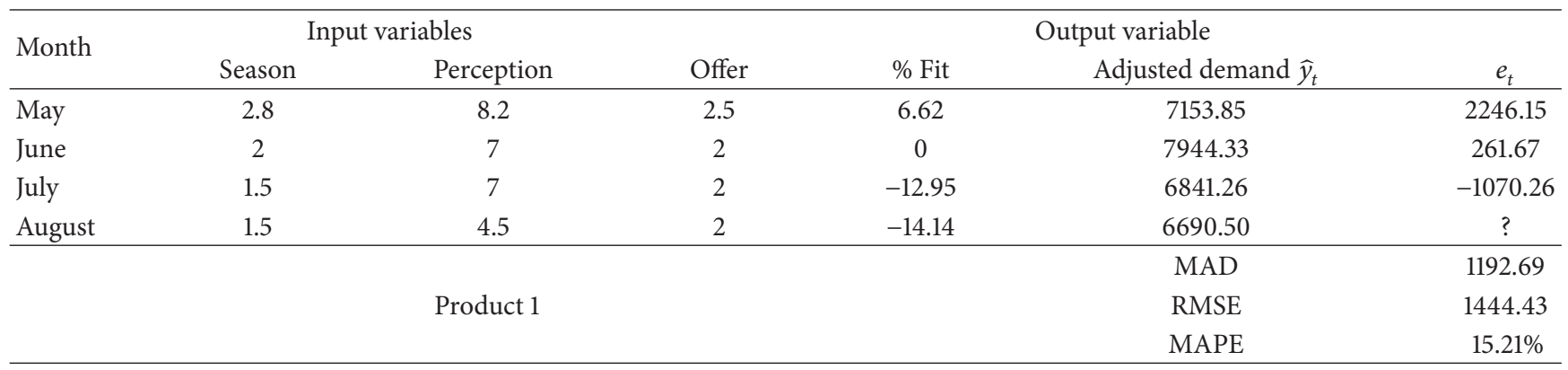

TABle 5: Adjusted demand product 2.

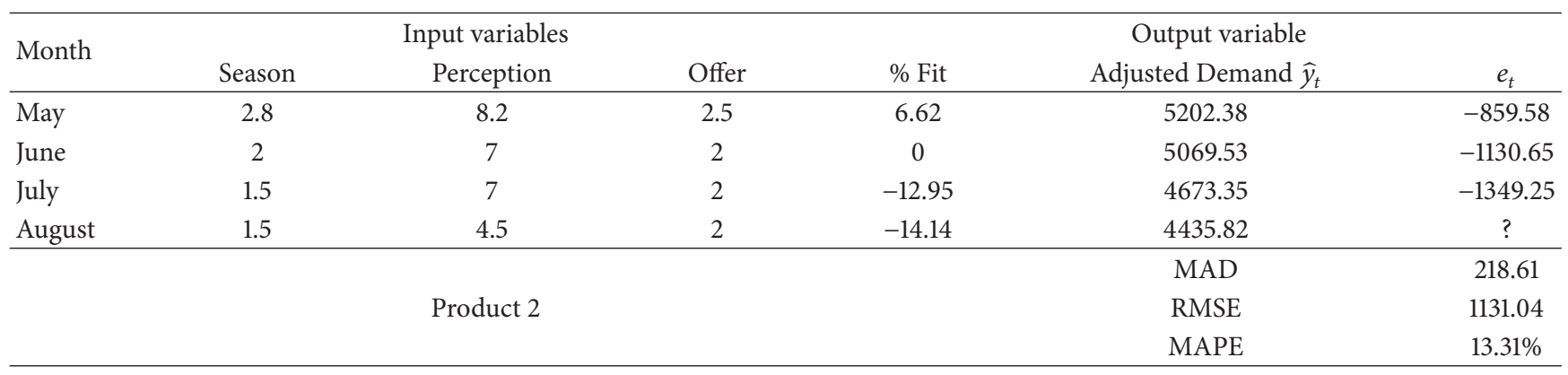

By having a reliable forecast of the demand for the three products, it was possible to best determine the daily quantity of products to manufacture and the cycle time. Finally this information represents the input data to the simulator. The results are shown in Table 7.

5.3. Synchronizing the Quantity to Order of Raw Material with Demand. The third step of the methodology was to synchronize the order quantity of raw material with the estimated demand. Figure 5 illustrates the relationship between the variables involved in the establishment of the order points and the safety stock. The main relationship is shown in (9) as follows:

$$
\mathrm{OP}=\mathrm{EDDLT}+\mathrm{SS}
$$

5.3.1. Fuzzy Model to Determine the Safety Stock. There are several methodologies to determine the safety stock. These techniques assume that the demand follows either a normal probability distribution or a discrete probability distribution. A fuzzy logic model allowed finding the safety stock taking into account the uncertainty.

The model included the following assumptions.

(i) The assumption of normality of daily demand was dismissed.

(ii) There are no contracts between the supplier and the company therefore there is no planning orders.

(iii) Sometimes it is difficult to find another supplier who can supply the product because either there are no competitors or the product's specifications do not permit it. (iv) There is no feedback of information between customer, company, and suppliers.

(1) Linguistic Variables and Fuzzy Sets. As in the case of the product demand, the amount of raw material that should be considered as safety stock was estimated by a fuzzy logic model considering the following factors: Delivery time, Quality and Shortage. Delivery time as the linguistic variable is defined as the fulfilling of the delivery date of the raw material by the supplier. Fuzzy sets that are part of this variable are three, which are: high, medium and low punctuality.

Quality is the next factor and as a linguistic variable is the term that refers to compliance with the specifications of raw materials stocked by the supplier. This variable considers three cases, and each of these cases defines a fuzzy set, which is good, fair, and poor quality.

The last factor to consider is the Shortage of raw materials. The shortage does not refer to the lack of material at the warehouse; it refers to the shortage of materials on the market in general. For these variable, three fuzzy sets are assumed: low, medium and serve shortage.

The output variable of the model represents an adjustment factor that will be multiplied by the demand during the lead time (EDDLT) to determine safety stock. This variable is named raw material. Under this consideration five cases are presented, which allow to define the fuzzy sets belonging to this variable: not critical, uncertain, critical, uncertaincritical, and supercritical. Figure 6 shows the sets of input variables and the output model for safety stock.

The summary of the rules used for the inference process is presented in Table 8 . 
TABLE 6: Adjusted demand product 3.

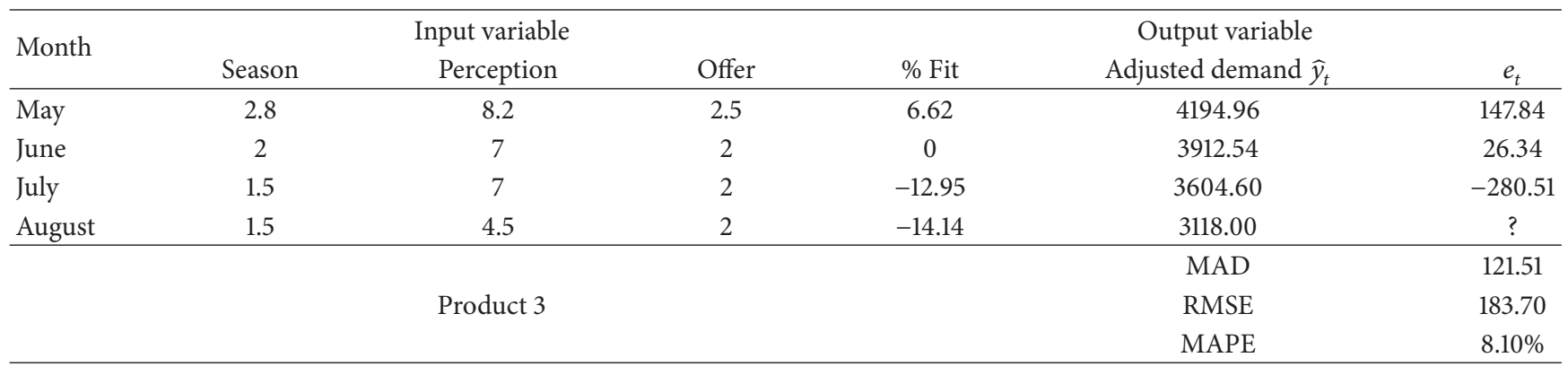

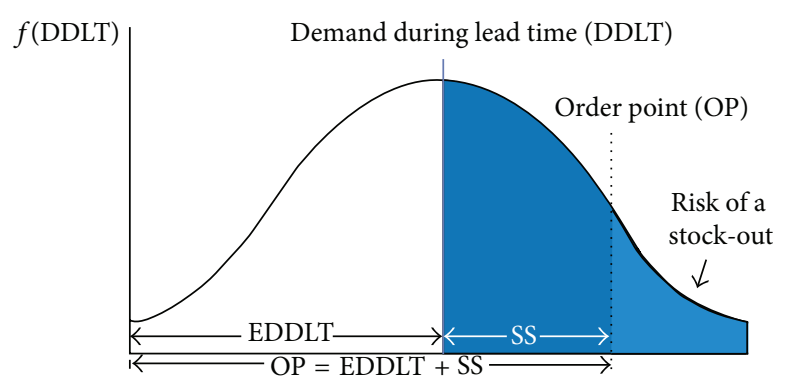

EDDLT: expected demand during lead time SS: safety stock

FIGURE 5: Relationship between DDLT, EDDLT, SS, OP and the probability of shortage of each cycle reorder.

TABLE 7: Simulation results according to the monthly demand for all three products.

\begin{tabular}{|c|c|c|c|c|c|}
\hline \multirow[t]{2}{*}{ Month } & \multirow{2}{*}{$\begin{array}{l}\text { Adjusted } \\
\text { demand for the } \\
\text { three products }\end{array}$} & \multicolumn{3}{|c|}{$\begin{array}{l}\text { Daily amount of pair of } \\
\text { shoes produced by each } \\
\text { type of product }\end{array}$} & \multirow[t]{2}{*}{$\begin{array}{l}\text { Line } \\
\text { efficiency }\end{array}$} \\
\hline & & 1 & 2 & 3 & \\
\hline May & 7154 & 265 & 208 & 168 & $85 \%$ \\
\hline June & 7944 & 294 & 203 & 157 & $95 \%$ \\
\hline July & 6841 & 253 & 187 & 144 & $81 \%$ \\
\hline August & 6691 & 248 & 178 & 125 & $79 \%$ \\
\hline
\end{tabular}

Where NC means not critical, INC means Unclear-not critical, C means Critical, IC means critical uncertain, and SC means super critical.

The fuzzy model will provide a percentage factor (variable output) that will be multiplied by the EDDLT to calculate the safety stock.

\subsection{Determine the Delivery Time: Fuzzy Model for the Product} Delivery Time. The products arrive to end consumers in two ways: to sell directly the products to shoe stores established in the region and to get their products through intermediaries, that is, their products are collected by a third company that store the products at central storehouses and distributes them to the point of final sale.

The model includes the following assumptions.

(i) There are no contracts between the supplier and the company; therefore there is no planning orders. (ii) There is no feedback of information between customer, company, and supplier.

(iii) The company is responsible for bringing products to point of sale.

5.4.1. Linguistic Variables and Fuzzy Sets. The two factors create uncertainty in the estimated delivery time: Ease of manufacturing product and the location of the point of sale. Ease of manufacturing as linguistic variable is defined as a facility, according to experience, that company has to satisfy the quantity of product requested by the customer. Fuzzy sets that are part of this variable are three: very possible, possible, and hardly possible. The location is the second factor and as a linguistic variable represent the distance between the factory and the place from which the order was placed. The variable considers two cases are near and far. The output variable of the model is an adjustment factor that multiplies the delivery time thrown by internal planning. This variable is named Fit. Under this consideration the 3 cases are presented, which allow to define the fuzzy sets belonging to this variable: the adjustment is null, slight, or large. Figure 7 shows the two input variables and the output of this model.

Finally Table 9 summarizing the rules used for the inference process.

The input data (fuzzy variables) were introduced to the fuzzy model and as results the model provided a percentage factor (variable output). This factor will be multiplied by the initial delivery time in order to give an accurate delivery date to the client. With these results the company is able to offer a delivery date where the breach of agreed orders is reduced. 

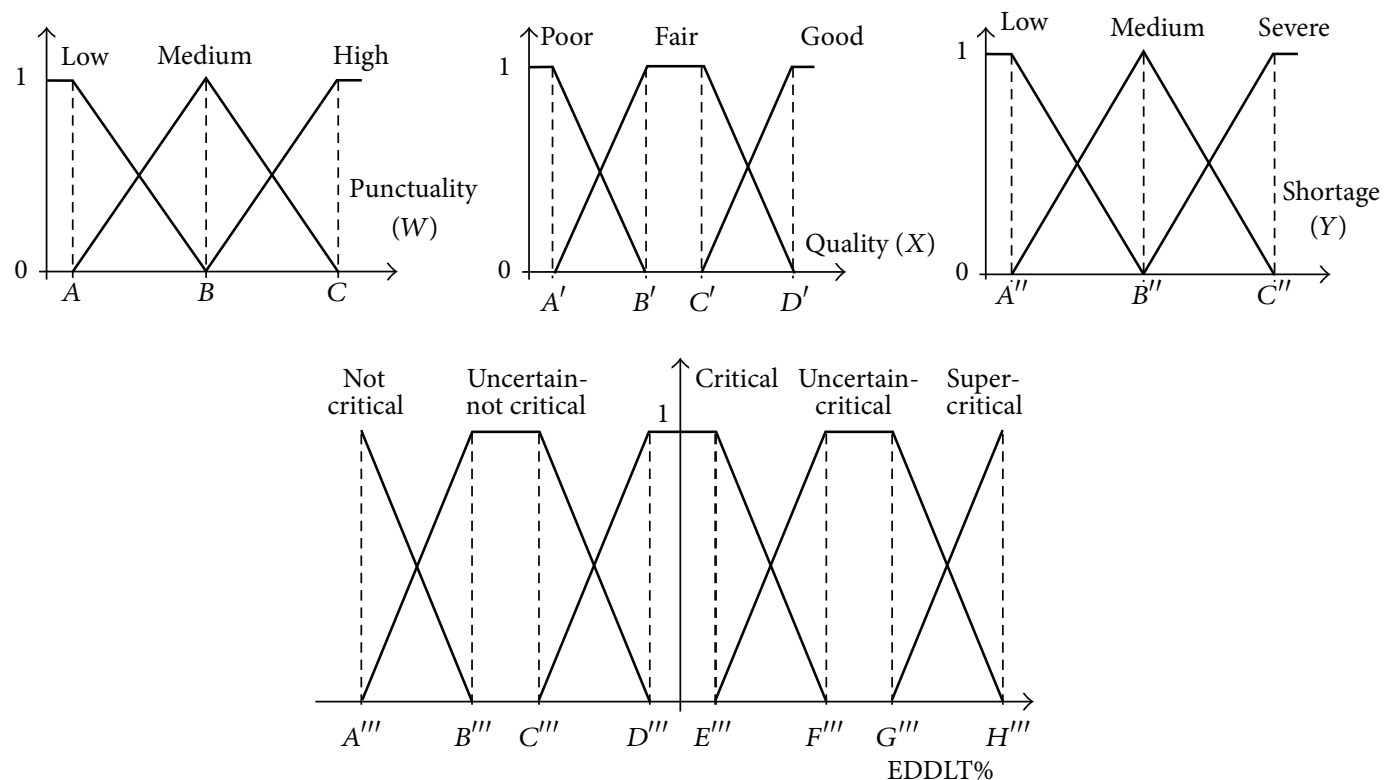

FIgURE 6: Fuzzy sets variable raw material.

TABLE 8: Fuzzy rules base.

\begin{tabular}{|c|c|c|c|c|c|c|c|c|c|}
\hline & \multicolumn{9}{|c|}{ Quality } \\
\hline & \multicolumn{3}{|c|}{ Poor } & \multicolumn{3}{|c|}{ Fair } & \multicolumn{3}{|c|}{ Good } \\
\hline & \multicolumn{3}{|c|}{ Shortage } & \multicolumn{3}{|c|}{ Shortage } & \multicolumn{3}{|c|}{ Shortage } \\
\hline & Low & Medium & Severe & Low & Medium & Severe & Low & Medium & Severe \\
\hline \multicolumn{10}{|l|}{ Punctuality } \\
\hline Low & $\mathrm{C}$ & SC & SC & $\mathrm{C}$ & UC & UC & UNC & UNC & $\mathrm{C}$ \\
\hline Medium & $\mathrm{C}$ & $\mathrm{C}$ & UC & $\mathrm{C}$ & $\mathrm{C}$ & UC & UNC & UNC & $\mathrm{C}$ \\
\hline High & UNC & C & UC & UNC & $\mathrm{C}$ & UC & NC & $\mathrm{NC}$ & UNC \\
\hline
\end{tabular}
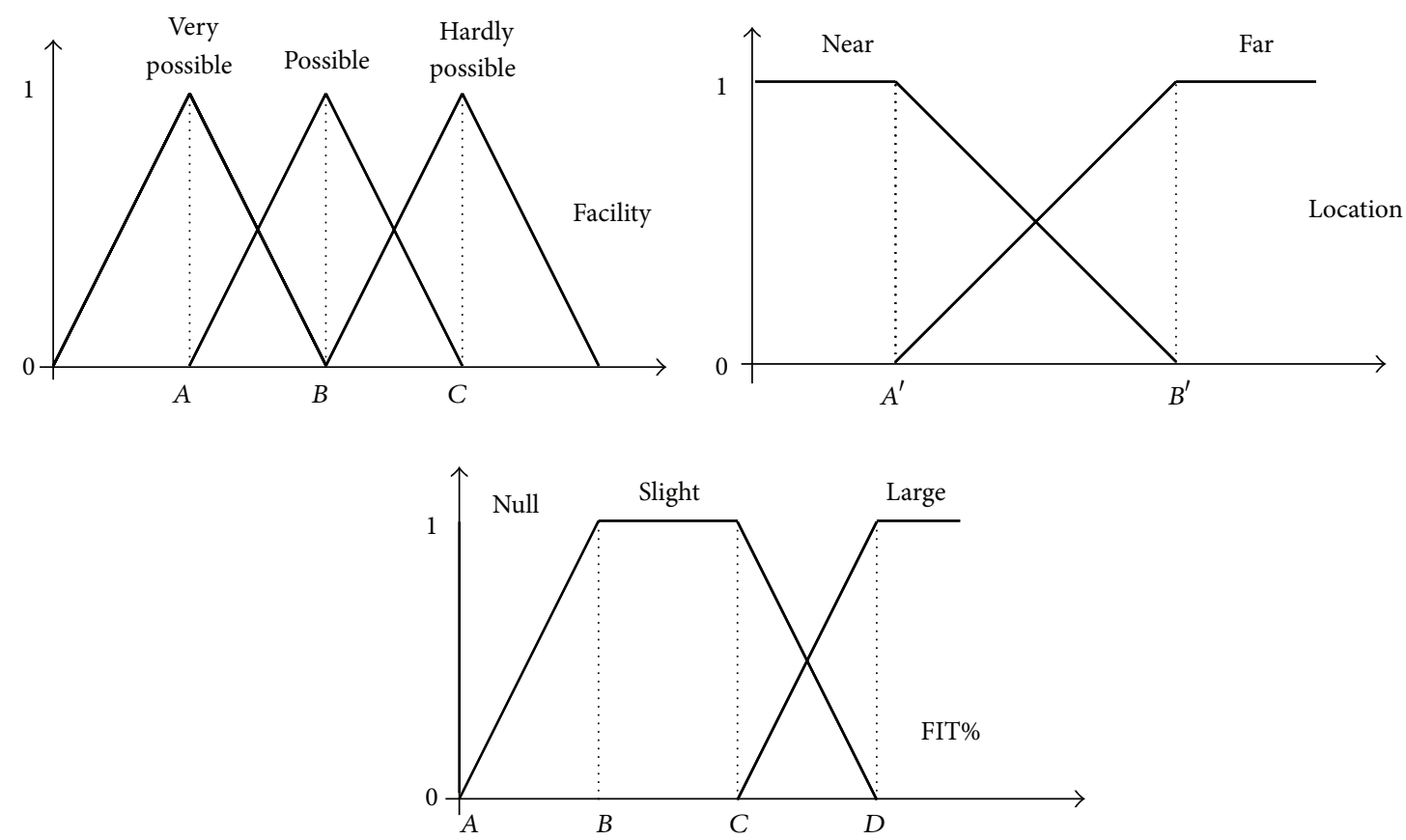

Figure 7: Fuzzy set of the output variable Fit. 


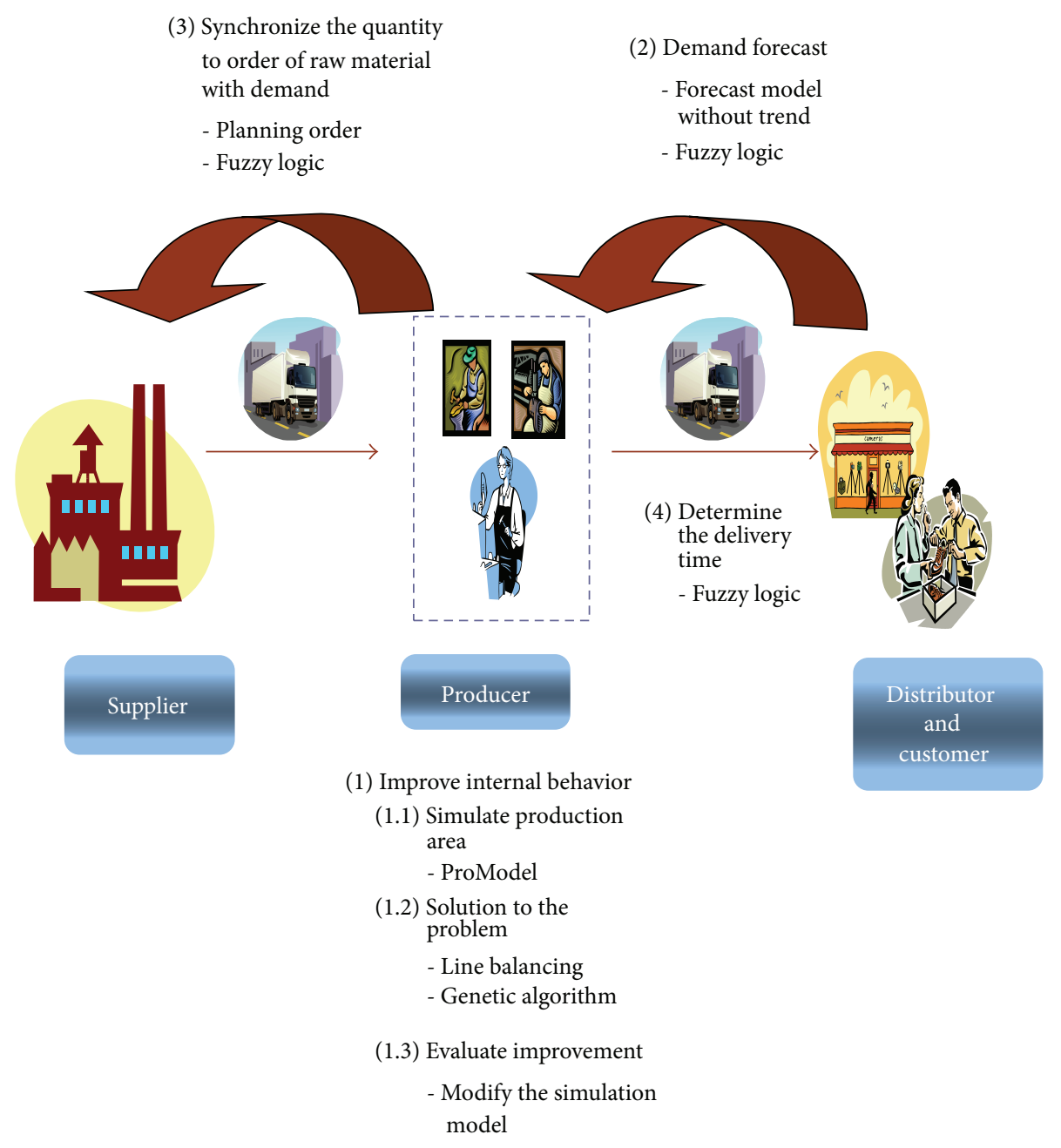

FIGURE 8: Methodology for the integration of the supply chain and techniques used in each step.

TABLE 9: Fuzzy rules base.

\begin{tabular}{lcc}
\hline & & Location \\
& Near & Far \\
\hline Easy of manufacturig & & \\
Very possible & Null & Slight \\
Possible & Slight & Large \\
Hardly possible & Slight & Large \\
\hline
\end{tabular}

A greater confidence between the producer and client will be established.

\section{Conclusions}

The SC integration is a major issue today, as it allows companies to position themselves in a highly competitive market. The main contribution of this research is to propose a sequence of logical steps to achieve this integration regardless of the nature of the company, especially when talking about companies that have manual processes.

As an advantage the proposed methodology permit to integrate the traditional engineering techniques with new developed tools as soft computing to overcome the problems that prevent the successful integration of the members of the SC adapting the use of them depending on the features and restrictions of each company without condition the use of any particular tool. This integration does not detract from the traditional methods of problem solving but complement each other. As already mentioned, the resulting models can be taken up to other companies. The application of this methodology in a company in the footwear industry shows its efficiency when it is applied to a business process which is considered artisanal and affected by an uncertain market. This approach helped the company to synchronize the SC to which it belongs. Figure 8 summarizes the methodology for the SC integration more broadly; in this scheme the techniques used for each of the steps in the methodology samples are mentioned.

\section{Conflict of Interests}

The authors declare that there is no conflict of interests regarding the publication of this paper. 


\section{References}

[1] S. A. Ghasimi, R. Ramli, and N. Saibani, "A genetic algorithm for optimizing defective goods supply chain costs using JIT logistics and each-cycle lengths," Applied Mathematical Modelling, vol. 38, no. 4, pp. 1534-1547, 2014.

[2] T. Efendigil and S. Önüt, "An integration methodology based on fuzzy inference systems and neural approaches for multi-stage supply-chains," Computers and Industrial Engineering, vol. 62, no. 2, pp. 554-569, 2012.

[3] L. L. Hon and W. P. Q. Ng, "A process integration approach for supply chain development," in Handbook of Process Integration (PI) Minimisation of Energy and Water Use, Waste and Emissions, Woodhead Publishing Series in Energy, pp. 571-593, 2013.

[4] INEGI, National Institute of Statistics, Geography and Informatics, 2013, http://www.inegi.org.mx.

[5] M. Fernando and C. F. Elisa, "Cómo administrar la cadena de suministro," 2008, http://www.cnnexpansion.com/ manufactura/actualidad/2008/03/26/como-administrar-lacadena-de-suministro.

[6] M. Paul, Supply Chain Analytics: What is it and Why is it so Important?, 2013, http://www.industryweek.com/blog/supplychain-analytics-what-it-and-why-it-so-important.

[7] M. L. Lengnick-Hall, C. A. Lengnick-Hall, and C. M. Rigsbee, "Strategic human resource management and supply chain orientation," Human Resource Management Review, vol. 23, no. 4, pp. 366-377, 2013.

[8] J. A. Palma-Mendoza, N. Kevin, and R. Rajat, "Business process re-design methodology to support supply chain integration," International Journal of Information Management, vol. 34, no. 2, pp. 167-176, 2014.

[9] A. A. Elimam and B. Dodin, "Project scheduling in optimizing integrated supply chain operations," European Journal of Operational Research, vol. 224, no. 3, pp. 530-541, 2013.

[10] H. Jafar, "Lead time variation control using reliable shipment equipment: an incentive scheme for supply chain coordination," Transportation Research Part E: Logistics and Transportation Review, vol. 63, pp. 44-58, 2014.

[11] S. Mehdi, "An integrated multi-objective model for allocating the limited sources in a multiple multi-stage lean supply chain," Economic Modelling, vol. 37, pp. 224-237, 2014.

[12] A. Zhang, H. Luo, and G. Q. Huang, "A bi-objective model for supply chain design of dispersed manufacturing in China," International Journal of Production Economics, vol. 146, no. 1, pp. 48-58, 2013.

[13] M. Khan, M. Y. Jaber, and A. Ahmad, "An integrated supply chain model with errors in quality inspection and learning in production," Omega, vol. 42, no. 1, pp. 16-24, 2014.

[14] T. Lin, F. Rodríguez Luis, N. Shastri Yogendra, C. Hansen Alan, and K. C. Ting, "Integrated strategic and tactical biomassbiofuel supply chain optimization," Bioresource Technology, vol. 156, pp. 256-266, 2014.

[15] Z. H. Zhang, B. F. Li, X. Qian, and L. N. Cai, "An integrated supply chain network design problem for bidirectional flows," Expert Systems with Applications, vol. 41, no. 9, pp. 4298-4308, 2014.

[16] Ö. Eren, P. Turan, and B. Tolga, "Modeling and optimizing the integrated problem of closed-loop supply chain network design and disassembly line balancing," Transportation Research Part E: Logistics and Transportation Review, vol. 61, pp. 142-164, January 2014.
[17] B. Sarkar and A. Majumder, "Integrated vendor-buyer supply chain model with vendor's setup cost reduction," Applied Mathematics and Computation, vol. 224, pp. 362-371, 2013.

[18] D. Song, J. Dong, and J. Xu, "Integrated inventory management and supplier base reduction in a supply chain with multiple uncertainties," European Journal of Operational Research, vol. 232, no. 3, pp. 522-536, 2014.

[19] J. Telhada, "Alternative MIP formulations for an integrated shift scheduling and task assignment problem," Discrete Applied Mathematics, vol. 164, part 1, pp. 328-343, 2014.

[20] D. Z. Zhang, "An integrated production and inventory model for a whole manufacturing supply chain involving reverse logistics with finite horizon period," Omega, vol. 41, no. 3, pp. 598-620, 2012.

[21] CANAICAL, "Chamber of the Footwear Industry of the State of Guanajuato," 2014, http://canaical.org/.

[22] C. T. Ragsdale and E. C. Brown, "On modeling line balancing problems in spreadsheets," INFORMS Transactions on Education, vol. 4, no. 2, 2004.

[23] J. Majumdar and A. K. Bhunia, "An alternative approach for unbalanced assignment problem via genetic algorithm," Applied Mathematics and Computation, vol. 218, no. 12, pp. 6934-6941, 2012.

[24] Ö. Mutlu, O. Polat, and A. A. Supciller, "An iterative genetic algorithm for the assembly line worker assignment and balancing problem of type-II," Computers and Operations Research, vol. 40, no. 1, pp. 418-426, 2013.

[25] N. E. Escobar Gómez, J. J. Díaz Núñez, and L. F. Taracena Sanz, "Model for adjustment of aggregate forecasts using fuzzy logic," Ingeniería Investigación y Tecnología, vol. 11, no. 3, pp. 289-302, 2010. 


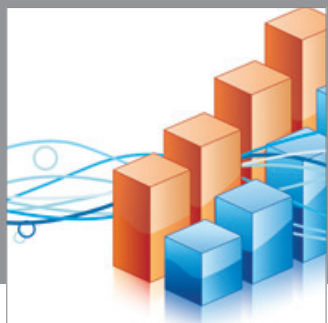

Advances in

Operations Research

mansans

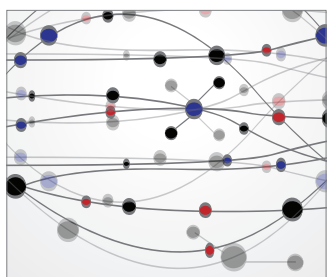

The Scientific World Journal
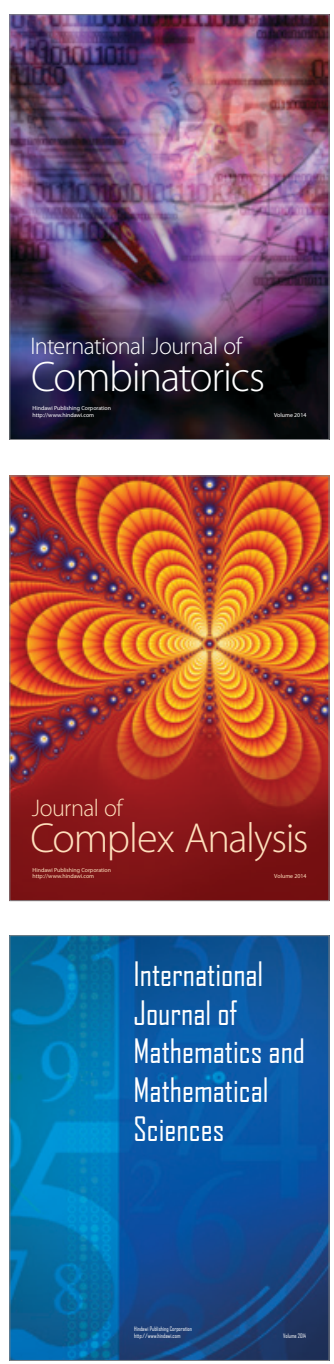
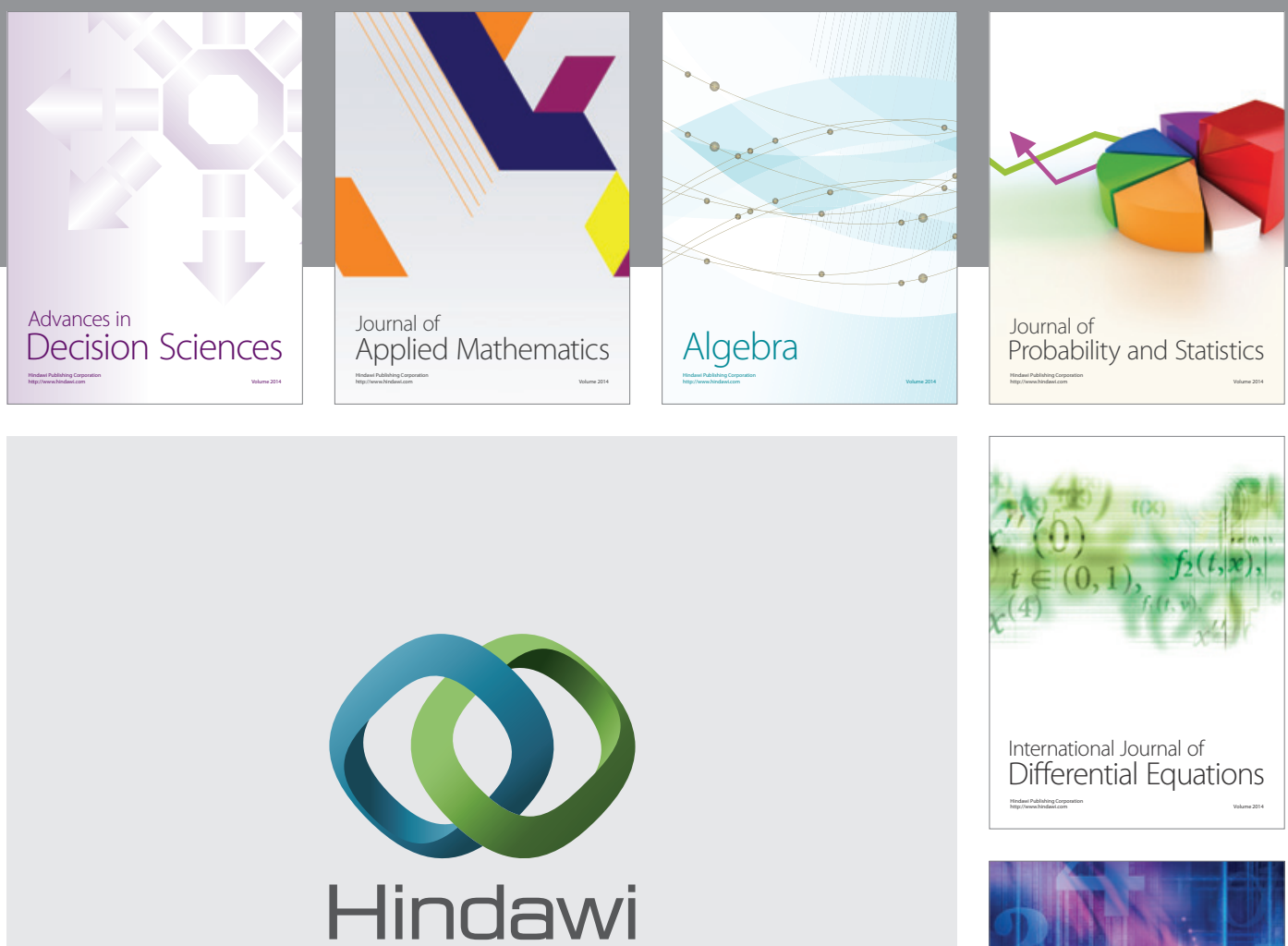

Submit your manuscripts at http://www.hindawi.com
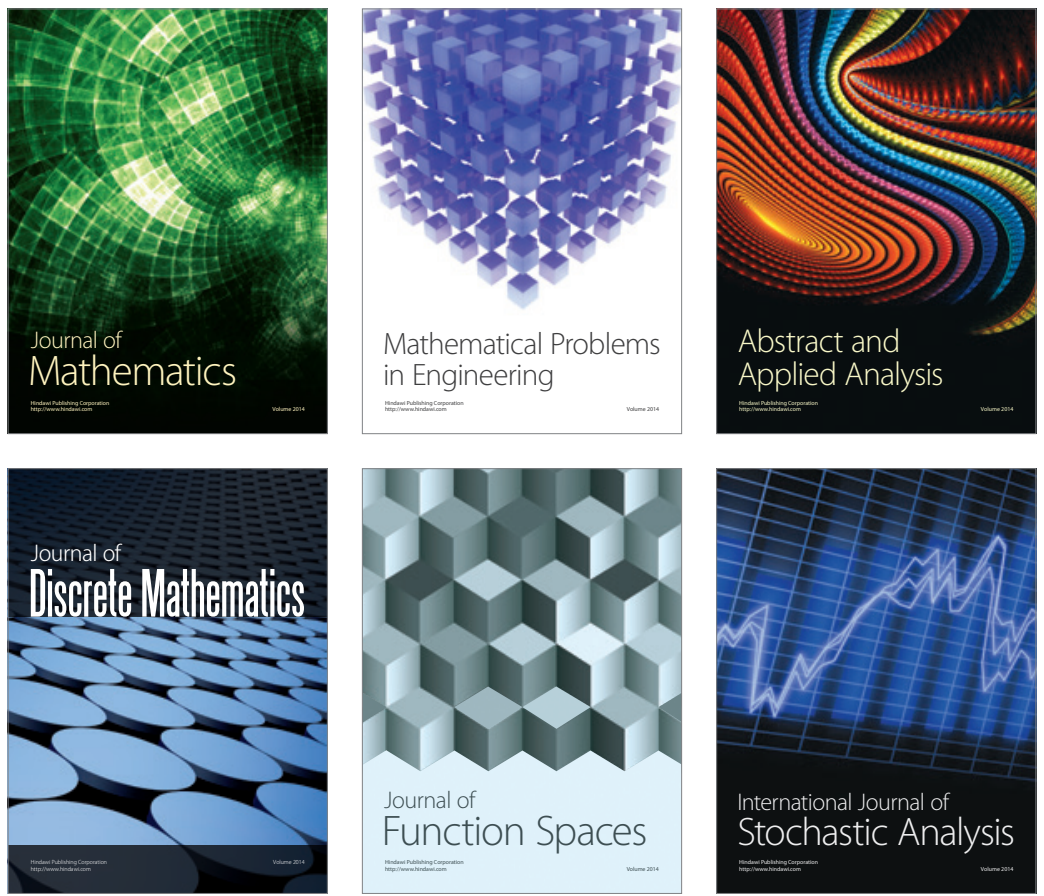

Journal of

Function Spaces

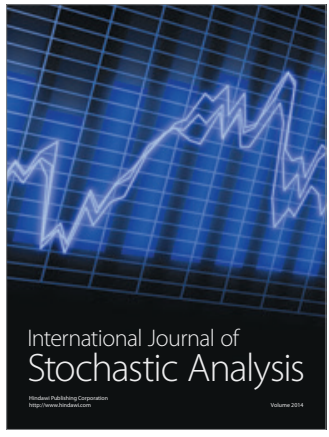

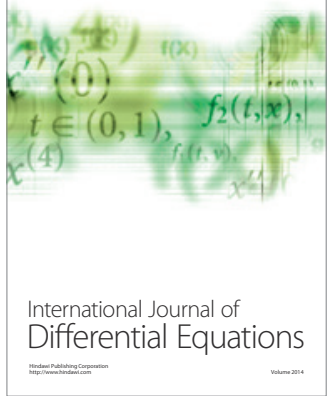
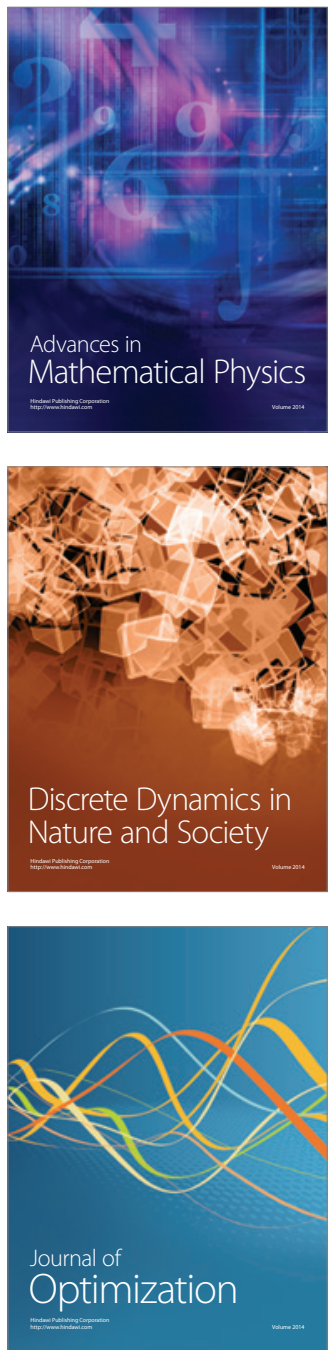